\title{
SÍNTESE DE NANOMATERIAIS UTILIZANDO GARRAFAS PET PÓS-CONSUMO COMO MATÉRIA-PRIMA
}

\author{
Joner Oliveira Alves 1 \\ Chuanwei Zhuo ${ }^{2}$ \\ Yiannis Angelo Levendis ${ }^{3}$ \\ Jorge Alberto Soares Tenório ${ }^{4}$
}

\section{Resumo}

Atualmente, são produzidas no Brasil mais de 12 milhões de unidades de garrafas PET por dia. As garrafas PET pós-consumo correspondem a cerca de I,0\% da produção dos resíduos sólidos urbanos. Neste trabalho, estuda-se o reaproveitamento de garrafas PET como matéria-prima para produção de nanomateriais. Amostras de garrafas PET pós-consumo foram inseridas em um forno elétrico com temperaturas de $600^{\circ} \mathrm{Ce} 1.000^{\circ} \mathrm{C}$, sob uma atmosfera de $15 \% \mathrm{O}_{2}$ e $85 \% \mathrm{~N}_{2}$. O efluente da queima foi submetido a um filtro de $\mathrm{SiC}$ e repassado a um segundo forno mantido a $1.000^{\circ} \mathrm{C}$. Telas de aço inoxidável foram dispostas no forno secundário para atuarem como catalisador. A técnica de microscopia eletrônica de varredura (MEV) foi empregada para a verificação da formação dos nanomateriais. Os resultados apontam a formação de nanomateriais com diâmetros de $90 \mathrm{~nm}$ a $140 \mathrm{~nm}$ e comprimentos na ordem de $40 \mu \mathrm{m}$. Os nanomateriais produzidos apresentam a forma tubular característica dos nanotubos de carbono.

Palavras-chave: PET; Nanomateriais; Combustão; Catálise.

\section{SYNTHESIS OF NANOMATERIALS USING POST-CONSUMER PET BOTTLES AS RAW MATERIAL}

\begin{abstract}
Currently, more than 12 million units of PET bottles are produced in Brazil per day. The post-consumer PET bottles correspond to about $1.0 \%$ of the production of urban solid wastes. In this work, the recovery of PET bottles as raw material to produce nanomaterials is studied. Samples of post-consumer PET bottles were inserted in an electric furnace with temperatures of 600 and $1000^{\circ} \mathrm{C}$ under an atmosphere of $15 \% \mathrm{O}_{2}$ and $85 \% \mathrm{~N}_{2}$. The burned effluent was subjected to a $\mathrm{SiC}$ filter and channeled into the second furnace at $1000^{\circ} \mathrm{C}$. Stainless steel meshes were placed in the second furnace in order to work as catalyst. Scanner electron microscopy (SEM) technique was used to verify the formation of nanomaterials. Results show the formation of nanomaterials with diameters of $90-140 \mathrm{~nm}$ and lengths of about $40 \mu \mathrm{m}$. Produced nanomaterials present the tubular form typical of carbon nanotubes.
\end{abstract}

Key words: PET; Nanomaterials; Combustion; Catalyst.

\section{INTRODUÇÃO}

O poli(tereftalato) de etileno - PET é um dos plásticos mais utilizados para a fabricação de garrafas, especialmente para bebidas carbonatadas. ${ }^{(1)}$ Formado pela reação entre o ácido tereftálico e o etileno glicol, esse polímero possui altas resistências mecânica e química, além de proporcionar uma barreira para gases e odores. ${ }^{(2)}$ De acordo com a National Association of PET Container Resources (Napcor) cerca de 2,4 milhões de toneladas de PET foram utilizadas em 2006 como matérias-primas para a produção de garrafas, sendo que este valor tende a aumentar anualmente. ${ }^{(3)}$

Apesar das diversas aplicações para o PET pós-consumo, $45 \%$ do total produzido ainda não é

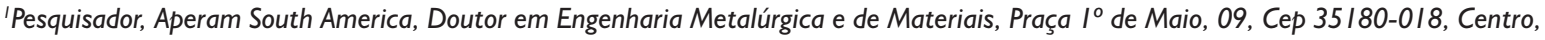

Timóteo, MG, Brasil. E-mail: joner.alves@aperam.com

2Doutorando, Northeastern University, Mestre em Engenharia Mecânica, 360 Huntington Av., Boston, MA, 02II5, USA.

E-mail: zhuo.c@husky.neu.edu

3Professor emérito, Northeastern University, Doutor em Engenharia Ambiental, 360 Huntington Av., Boston, MA, 02II5, USA.

E-mail: y.levendis@neu.edu

${ }^{4}$ Professor titular, Doutor em Engenharia Metalúrgica, Universidade de São Paulo - USP, Av. Prof. Mello Moraes, 2463, Cep 05508-030,

Cidade Universitária, São Paulo, SP, Brasil. E-mail: jtenorio@usp.br
} 
reaproveitado. A redução do volume de materiais plásticos destinados a aterros sanitários é importante, pois proporciona melhorias no processo de decomposição da matéria orgânica, uma vez que o plástico impermeabiliza as camadas em decomposição, prejudicando a circulação de gases e líquidos. No Brasil, a produção de PET é equivalente a cerca de $9,0 \%$ da produção total de plásticos; contudo, a fração de PET no resíduo sólido urbano corresponde, em média, a 20\% do total. Esta discrepância é justificada pelo fato de plásticos com maior consumo (como PP e PVC) serem empregados na fabricação de produtos com longa vida útil (como nas indústrias de bens de consumo e construção civil), enquanto o PET é usado majoritariamente em embalagens (produtos com curta vida útil) ${ }^{(3,4)}$

Uma opção para a destinação final do PET pós-consumo é a combustão com aproveitamento energético. Nesse processo, o material é queimado em atmosfera controlada visando a geração de vapor e energia elétrica. Garrafas plásticas possuem potencial energético de 22-25 MJ/kg e, quando controladamente queimadas, podem produzir energia com baixos índices de poluição. ${ }^{(5,6)}$ Neste trabalho é estudada a adição de um sistema catalisador ao processo de combustão do PET visando uma recuperação dos gases gerados na forma de nanomateriais.

A nanotecnologia tem despertado o interesse de diversas áreas devido às enormes possibilidades de aplicações. Os nanomateriais são materiais com graus estruturais na ordem de nanometros $\left(\mathrm{IO}^{-9} \mathrm{~m}\right)$, ou seja, de um bilionésimo de metro. ${ }^{(7)}$ Diversos tipos de nanomateriais, como os nanotubos de carbono, nanopartículas metálicas, nanopartículas de óxidos de metais de transição, nanoemulsões e diferentes tipos de nanocompósitos, deixaram de serem apenas alvos dos laboratórios acadêmicos e podem facilmente ser encontrados em produtos comercializados atualmente. Em 2010, os produtos que empregam a nanotecnologia movimentaram mundialmente cerca de US\$ I I trilhões, sendo que US\$ 340 bilhões foram correspondentes apenas aos nanomateriais, por exemplo, os catalisadores para automóveis, materiais para gravação magnética e componentes para protetores solares. ${ }^{(8,9)}$

\section{MATERIAIS E MÉTODOS}

Os materiais empregados neste trabalho foram embalagens de garrafas de bebidas feitas inteiramente de PET coletadas do resíduo urbano convencional. A Tabela I exibe a composição química desse resíduo.

As garrafas PET foram lavadas e cortadas manualmente em pedaços de aproximadamente $10 \mathrm{~mm}^{2}$. A Figura I exibe uma amostra do material utilizado nos experimentos.
Tabela I. Análise química do resíduo empregado (em \%p)

\begin{tabular}{cccc}
\hline $\mathbf{C}$ & $\mathbf{O}$ & H & Outros \\
\hline 62,6 & 31,8 & 4,7 & 0,9 \\
\hline
\end{tabular}

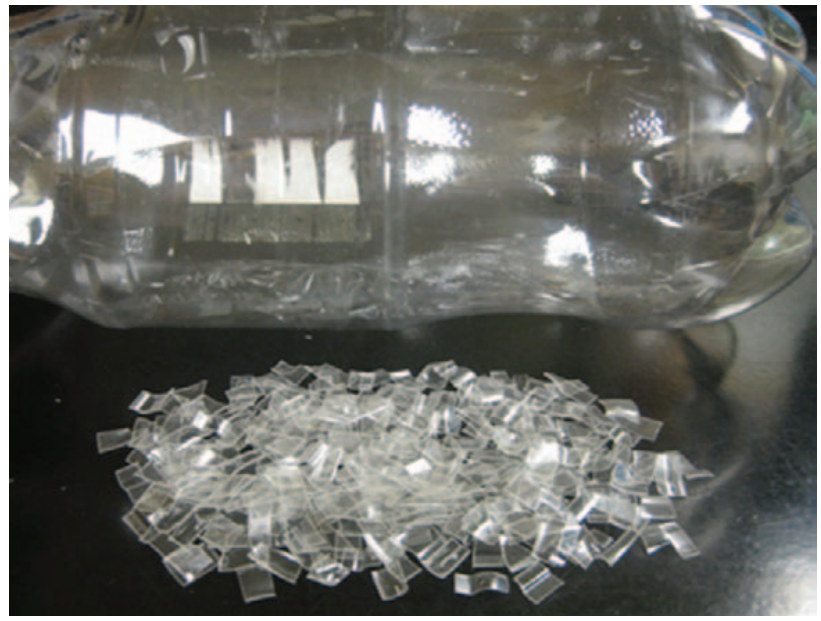

Figura I. Amostras de garrafas PET utilizadas.

Um cadinho de cerâmica foi utilizado para inserir $4,0 \mathrm{~g}$ de garrafas PET no sistema. $O$ aparato empregado consiste em dois fornos tubulares em escala laboratorial, ligados por um tubo de quartzo. Os fornos possuem capacidade de aquecimento independente e podem atingir I. $100^{\circ} \mathrm{C}$. Um desenho esquemático do aparato pode ser visto na Figura 2.

Visando a ausência inicial de ar, a atmosfera do aparato foi controlada com a adição de nitrogênio na entrada do tubo de quartzo. $O$ cadinho foi inserido no forno primário juntamente com a adição de jatos de oxigênio, criando uma atmosfera de combustão com $15 \%$ de $\mathrm{O}_{2}$ e $85 \%$ de $\mathrm{N}_{2}$. Foram testadas temperaturas do forno de combustão de $600^{\circ} \mathrm{C}$ e $1.000^{\circ} \mathrm{C}$. O produto da queima foi submetido a um filtro de carbeto de silício (SiC) que retém $97 \%$ das partículas submicrométricas. O efluente gasoso foi direcionado ao forno secundário pré-aquecido a $1.000^{\circ} \mathrm{C}$, onde foi colocado o catalisador.

O sistema catalisador empregado neste trabalho consistiu em um aço inoxidável do tipo AISI 304, apresentado na forma de uma tela vazada com 157 fios $/ \mathrm{cm}^{2}$ e diâmetros de $0,02 \mathrm{~mm}$. A tela foi utilizada como rece-

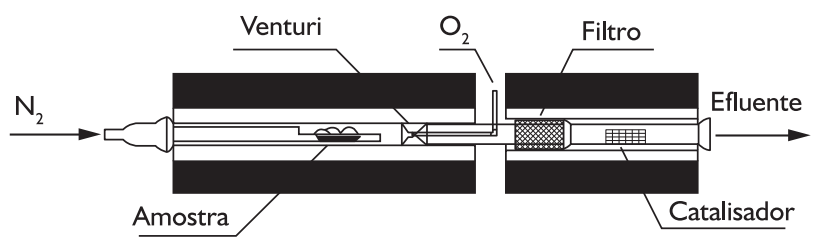

Figura 2. Desenho esquemático do aparato utilizado. 
bida (sem pré-tratamentos), tendo apenas sido realizada a limpeza com acetona antes de cada experimento. A Tabela 2 apresenta a especificação dos componentes de liga para o aço inoxidável 304 de acordo com a norma ASTM E2016. ${ }^{(10)}$

Amostras de diferentes regiões da tela metálica foram recortadas, embutidas e analisadas em um Microscópio Eletrônico de Varredura (MEV) modelo Hitachi 4800, com uma tensão de aceleração de $3 \mathrm{kV}$ e distância de trabalho de $8,2 \mathrm{~mm}$, localizado na Northeastern University (Boston, EUA).
Tabela 2. Componentes de liga (em \%p) para o aço inoxidável 304 de acordo com a norma ASTM E20 I $6^{(10)}$

\begin{tabular}{cccccccc}
\hline $\mathbf{C r}$ & $\mathbf{N i}$ & $\mathbf{M n}$ & $\mathbf{S i}$ & $\mathbf{N}$ & $\mathbf{C}$ & $\mathbf{P}$ & $\mathbf{S}$ \\
\hline $18,0-20,0$ & $8,0-10,5$ & $\leq 2,0$ & $\leq 1,0$ & $\leq 0,1$ & $\leq 0,08$ & $\leq 0,05$ & $\leq 0,03$ \\
\hline
\end{tabular}

\section{RESULTADOS E DISCUSSÕES}

Os resultados da análise por microscopia eletrônica de varredura (MEV) dos materiais sintetizados, a partir da combustão de garrafas PET pós-consumo, estão exibidos
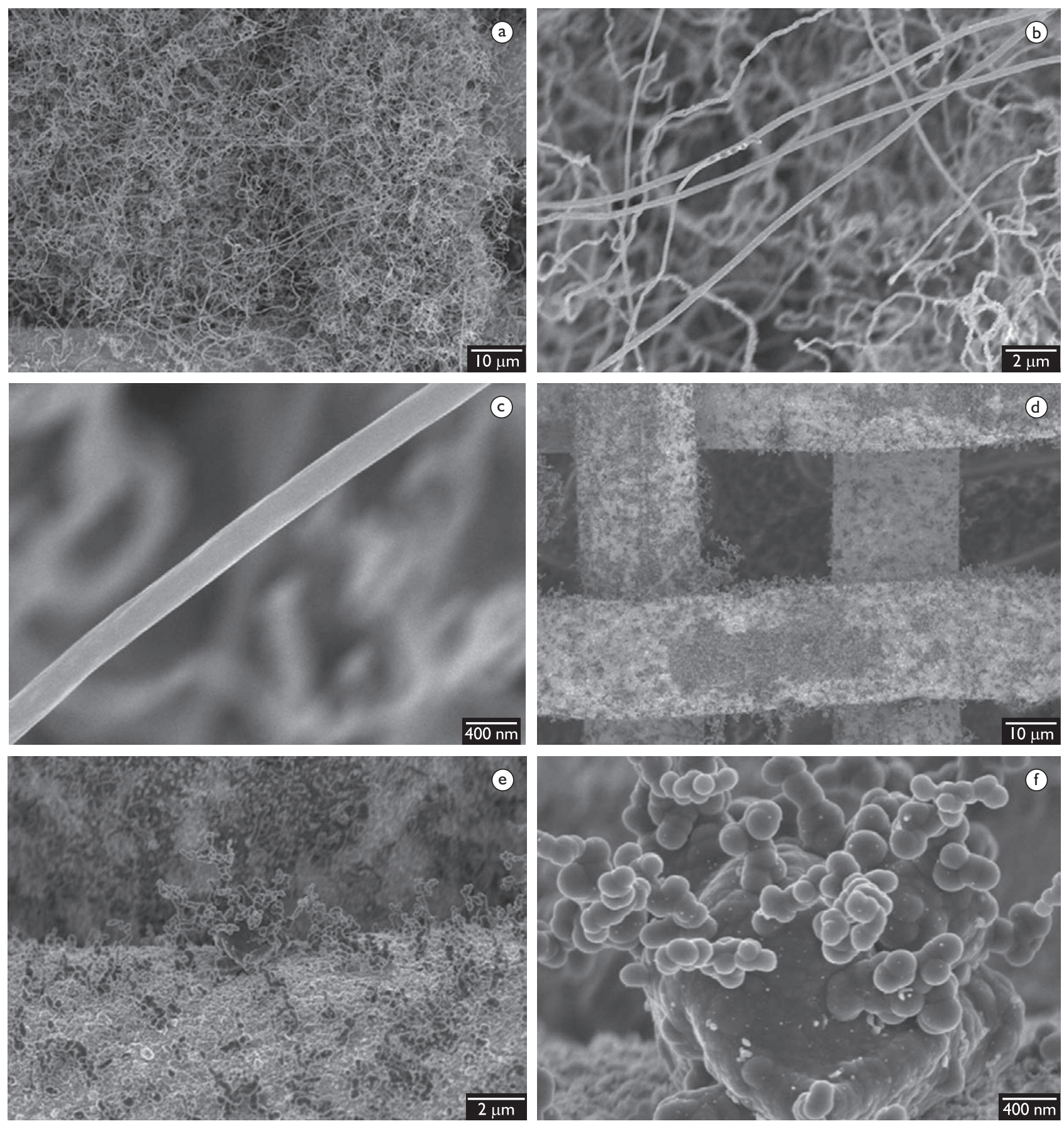

Figura 3. Imagens geradas pela técnica MEV dos nanomateriais obtidos com a combustão de garrafas $\mathrm{PET}$ a $600^{\circ} \mathrm{C}(\mathrm{a}, \mathrm{b}, \mathrm{c})$ e $1.000^{\circ} \mathrm{C}(\mathrm{d}, \mathrm{e}, \mathrm{f})$.

Tecnol. Metal. Mater. Miner., São Paulo, v. 9, n. I, p. 59-63, jan.-mar. 2012 
na Figura 3. As imagens a-c (Figura 3) exibem os materiais sintetizados com gases gerados por uma queima a $600^{\circ} \mathrm{C}$, enquanto que as imagens $\mathrm{d}$-f (Figura 3 ) representam produtos da queima a $1.000^{\circ} \mathrm{C}$.

Os resultados evidenciam a formação de nanomateriais nos experimentos que tiveram a temperatura de combustão de $600^{\circ} \mathrm{C}$. Um emaranhado de fibras com diâmetros entre $90 \mathrm{~nm}$ e $140 \mathrm{~nm}$ e comprimentos na ordem de $40 \mu \mathrm{m}$ foram formados de modo não uniforme sob a tela catalisadora, como pode ser observado nas imagens 3a-c (Figura 3).

A forma tubular apresentada pelos nanomateriais obtidos com a temperatura de combustão de $600^{\circ} \mathrm{C}$ é característica dos nanotubos de carbono (CNTs). Os nanotubos são os mais representativos materiais que podem ser enquadrados na classe dos nanomateriais. Os CNTs são constituídos de uma ou várias folhas de grafeno enroladas de forma concêntrica e com a cavidade interna oca. Esses materiais possuem inúmeras possibilidades de aplicações tecnológicas devido às suas excepcionais propriedades, tais como resistência química, baixa massa específica, resistências à oxidação e à temperatura, transporte elétrico, elevadas resistências mecânica e à ruptura, além de flexibilidade. (7)

Os experimentos realizados com temperatura de queima de $1.000^{\circ} \mathrm{C}$ não apresentaram formação de nanomateriais, tendo sido observada a presença de estruturas características do negro de fumo, como apresentado nas imagens 3d-f (Figura 3).

Diferentes mecanismos de crescimento baseados em observações experimentais e em cálculos teóricos foram propostos na literatura para explicar a formação e crescimento dos nanomateriais. Entretanto, o mecanismo exato pelo qual os nanomateriais são formados ainda não está completamente estabelecido. Baseando-se na teoria de Vapor-Liquid-Solid (VLS) para crescimento de cristais, proposta por Wagner e Ellis, ${ }^{(1)}$ Baker et al. ${ }^{(12)}$ e Baker ${ }^{(13)}$ estudaram o crescimento de fibras de carbono através da decomposição catalítica de fontes de hidrocarbonetos nas superfícies ativas de metais de transição. Durante esse processo, os hidrocarbonetos são decompostos em carbono e hidrogênio, seguido por uma difusão do carbono sobre a superfície do metal, até a solução tornar-se saturada. A supersaturação da solução resulta na precipitação de partículas sólidas de carbono na superfície do metal.

A combustão de garrafas PET pós-consumo gera hidrocarbonetos leves, principalmente, na forma de metano, etileno, acetileno e benzeno. ${ }^{(4,14)}$ Estes gases constituíram a fonte de carbono para a formação dos nanomateriais apresentados neste trabalho. ${ }^{(9,15)}$

\section{CONCLUSÕES}

Os hidrocarbonetos leves liberados pela queima das garrafas PET pós-consumo podem ser utilizados como fonte de carbono para a produção de nanomateriais, proporcionando uma destinação rentável para esses resíduos. Para uma temperatura de combustão de $600^{\circ} \mathrm{C}$ foi observada a formação de um emaranhado de nanomateriais com diâmetros entre $90-140 \mathrm{~nm}$ e comprimentos na ordem de $40 \mu \mathrm{m}$. Os nanomateriais formados remetem à forma tubular característica dos nanotubos de carbono, materiais com ampla gama de aplicações devido as suas excelentes propriedades.

\section{Agradecimentos}

Os autores agradecem ao CNPq pelo financiamento do período do Dr. Joner Alves, na Northeastern University (EUA), e ao suporte financeiro concedido pela CAPES (Edital 04/Cll-2008 - Rede Nanobiotec-Brasil).

\section{REFERÊNCIAS}

I HOPEWELL, J.; DVORAK, R.; KOSIOR, E. Plastics recycling: challenges and opportunities. Philosophical Transactions of the Real Society B: Biological Sciences, v. 364, n. 1526, p. 2 I I5-26, 2009. PMid:19528059. PMCid:2873020. http:// dx.doi.org/I0.1098/rstb.2008.03।I

2 MANCINI, S. D.; ZANIN, M. Influência de meios reacionais na hidrólise de PET pós-consumo. Polímeros: Ciência e Tecnologia, v. I2, n. I, p. 34-40, 2002.

3 NAPCOR. Report on post consumer PET container recycling activity. National Association for PET Container Resources. Disponível em: <http://www.napcor.com/PET/landing_petrecycling.html>. Acesso em: 20 set. 2010.

4 ALVES, J. O. et al. Caracterização das emissões de hidrocarbonetos leves geradas durante o processo de combustão de garrafas PET. In: CONGRESSO ANUAL DA ABM, 66., 20I I, São Paulo. Anais...São Paulo: ABM, 201 I. p. $1584-9 \mid$.

5 GONCALVES, C. K. et al. Emissions from the premixed combustion of gasified polyethylene. Energy Fuels, v. 22, n. I, p. 372-8I, 2007. http://dx.doi.org//0.1021/ef700379c

6 REA. Determination of biomass energy content of fuels. Renewable Energy Association. Disponível em: <http:// www.ftc.gov/os/comments/carbonworkshop/533254-000 I4.pdf>. Acesso em: 10 out. 2010.

7 ZARBIN, A. J. G. Química de (nano)materiais. Química Nova, v. 30, n. 6, p. I484-90, 2007. 
8 PITKETHLY, M. J. Nanoparticles as building blocks. Nano Today, v. 36, p. 36-42, 2003.

9 ALVES, J. O. Síntese de nanotubos de carbono através do uso do bagaço da cana-de-açúcar como matéria-prima. In: UNESCO, MBC, RECyT/ MERCOSUL, CNPq. (Org.). Nanotecnologia para o MERCOSUL. edição 2010 do prêmio Mercosul de Ciência e Tecnologia. Brasília: Unesco, 20I0. v. I, p. 57-74.

I0 ASTM INTERNATIONAL. ASTM E2016 - 06 Standard specification for industrial woven wire cloth. West Conshohocken, PA; 2006.

I I WAGNER, R. S.; ELLIS, W. C. Vapor-liquid-solid mechanism of single crystal growth. Applied Physics Letters, v. 4, p. 89-90, 1964. http://dx.doi.org//0.1063/1.1753975

12 BAKER, R. T. K. et al. Formation of filamentous carbon from iron, cobalt and chromium catalyzed decomposition of acetylene. Journal of Catalysis, v. 30, n. I, p. 86-95, 1973. http://dx.doi.org/10.1016/002I-9517(73)90055-9

13 BAKER, R. T. K. Catalytic growth of carbon filaments. Carbon, v. 27, n. 3, p. 315-23, 1989. http://dx.doi. org/10.1016/0008-6223(89)90062-6

I4 ALVES, J. O. et al. Analysis and control of light hydrocarbon gases in the pyrolysis-combustion process of several solid wastes. In: TMS ANNUAL MEETING \& EXHIBITION, I40., 20II, San Diego - EUA. Proceedings... Warrendale: EPD Congress, 20I I. I CD-ROM.

I5 ALVES, J. O. et al. Catalytic conversion of wastes from the bioethanol production into carbon nanomaterials. Applied Catalysis B: Environmental, v. I06, n. 3-4, p. 433-44, 201 I. http://dx.doi.org/I0.1016/j.apcatb.20I I.06.00 I

Recebido em: 03/10/20II

Aceito em: 08/02/2012 